\title{
Session III
}

\section{Variable Winds}

chair: A.W. Fullerton, N. Markova and D. Massa 


\title{
O-Star Wind Variability in the Ultraviolet and Optical Range
}

\author{
Lex Kaper
}

Astronomical Institute, University of Amsterdam, Kruislaan 403, 1098 Amsterdam, The Netherlands

\begin{abstract}
Variability is a fundamental property of O-star winds. One of the major breakthroughs in this field of research has been the recognition that wind variability is cyclical in nature. This suggests that stellar rotation is an important piece in the unsolved puzzle of the wind-variability mechanism. The current idea is that Corotating Interacting Regions (CIRs) are responsible for the observed wind variability. These conclusions are based on detailed and extensive monitoring of ultraviolet resonance lines formed throughout the stellar wind, and subordinate lines like $\mathrm{H} \alpha$ which probe the regions close to the star.
\end{abstract}

\section{Introduction}

The P Cygni profiles of strong ultraviolet resonance lines (e.g., of N v, Si IV, C rv) have proven to be a powerful tool in the study of the supersonically expanding winds of hot stars. The blue-shifted absorption troughs trace the wind material in front of the star, allowing a direct measurement of the velocity distribution of the accelerating plasma. The most prominent features of wind variability are the so-called discrete absorption components (DACs), which migrate through the profile from red to blue on a timescale of hours to days. Obviously, DACs cannot be observed in saturated P Cygni profiles; however, the steep blue edges of these profiles often show regular shifts of up to $10 \%$ in velocity. Edge variability is most probably related to the DAC behaviour, but the precise phase relation has not been unraveled yet. The P Cygni emission, centered at rest wavelength, is constant with time. This is what one would expect in the case of relatively modest variations in the stellar-wind structure. However, the intervening material has to cover a significant fraction of the stellar disk in order to give rise to a noticeable change in P Cygni absorption.

Because of their specific shape DACs are readily recognized in single snapshot spectra. In ultraviolet spectra obtained with the Ultraviolet International Explorer (IUE), Howarth \& Prinja (1989) detected DACs in more than $80 \%$ of a sample of 203 galactic $O$ stars. Taking into account that saturation of $\mathrm{P}$ Cygni profiles in $\mathrm{O}$ stars with very dense stellar winds prohibits the detection of DACs, one must conclude that the occurrence of DACs is a fundamental property of $\mathrm{O}$-star winds. 
The key problem is to understand why DACs start to develop and how they evolve. Extensive monitoring campaigns with the IUE satellite (e.g. Prinja et al. 1992, Howarth et al. 1993, Massa et al. 1995 (MEGA campaign), Kaper et al. 1996, 1998a) have resulted in a detailed quantitative description of wind variability in about a dozen bright $\mathrm{O}$ stars. For a given star, the DAC behaviour results in a characteristic variability pattern, which undergoes detailed changes from year to year.

In search for the origin of DACs, spectral lines of different strength can be used to probe the stellar wind at various depths. The UV resonance lines form throughout the stellar wind up to the outer regions where the wind has reached its terminal velocity. Strong subordinate lines (like N IV $1718 \AA$ and $\mathrm{H} \alpha$ ) are formed closer to the star where the density is higher, because the population of the lower level of the transition (which is not the ground state) is proportional to $\rho^{2}$. Monitoring of these lines would tell us whether wind variability sets in already close to the star. A complication is that both emission and absorption changes might contribute to the variability, and projection effects have to be taken into account. Whether or not the formation of DACs is triggered by variations at the photospheric level has to be investigated with help of high-resolution (both in wavelength and time) spectroscopy of deep photospheric lines. Therefore, coordinated ultraviolet and optical spectroscopic monitoring is required to study the relationship between variability in different lines in order to reveal the eventual "photospheric connection".

\section{Quantitative analysis DAC behaviour}

A detailed analysis of time series of ultraviolet spectra from a sample of 10 bright O stars has been presented by Kaper et al. (1996, 1998a). The migrating DACs are isolated from the underlying P Cygni profiles using a least-absorption template constructed from the datasets. Fits with exponential gaussian functions, with the known doublet separation and ratio in oscillator strength as fixed parameters, yield DAC parameters like the central velocity and column density, which are studied as a function of time. The DACs start at low velocity as broad absorption features and accelerate, while narrowing, until an asymptotic velocity $\left(v_{\text {asymp }}\right)$ is reached. This velocity is systematically lower (by 10-20\%) than the maximum "blue edge velocity" observed in saturated P Cygni lines, which has historically been identified with the terminal velocity, $v_{\infty}$, of the stellar wind. The proposed solution is that $v_{\text {asymp }}$ measures the "real" $v_{\infty}$ and that the additional blueshift in saturated P Cygni lines is caused by small-scale structure in the stellar wind (e.g. due to shocks resulting from the instability of radiation-driven winds, cf. Owocki, Feldmeier, this volume). In some stars, $v_{\text {asymp }}$ systematically changes from event to event. Taking into account optical depth effects, this might explain the gradual changes observed in the steep edge in saturated profiles. 

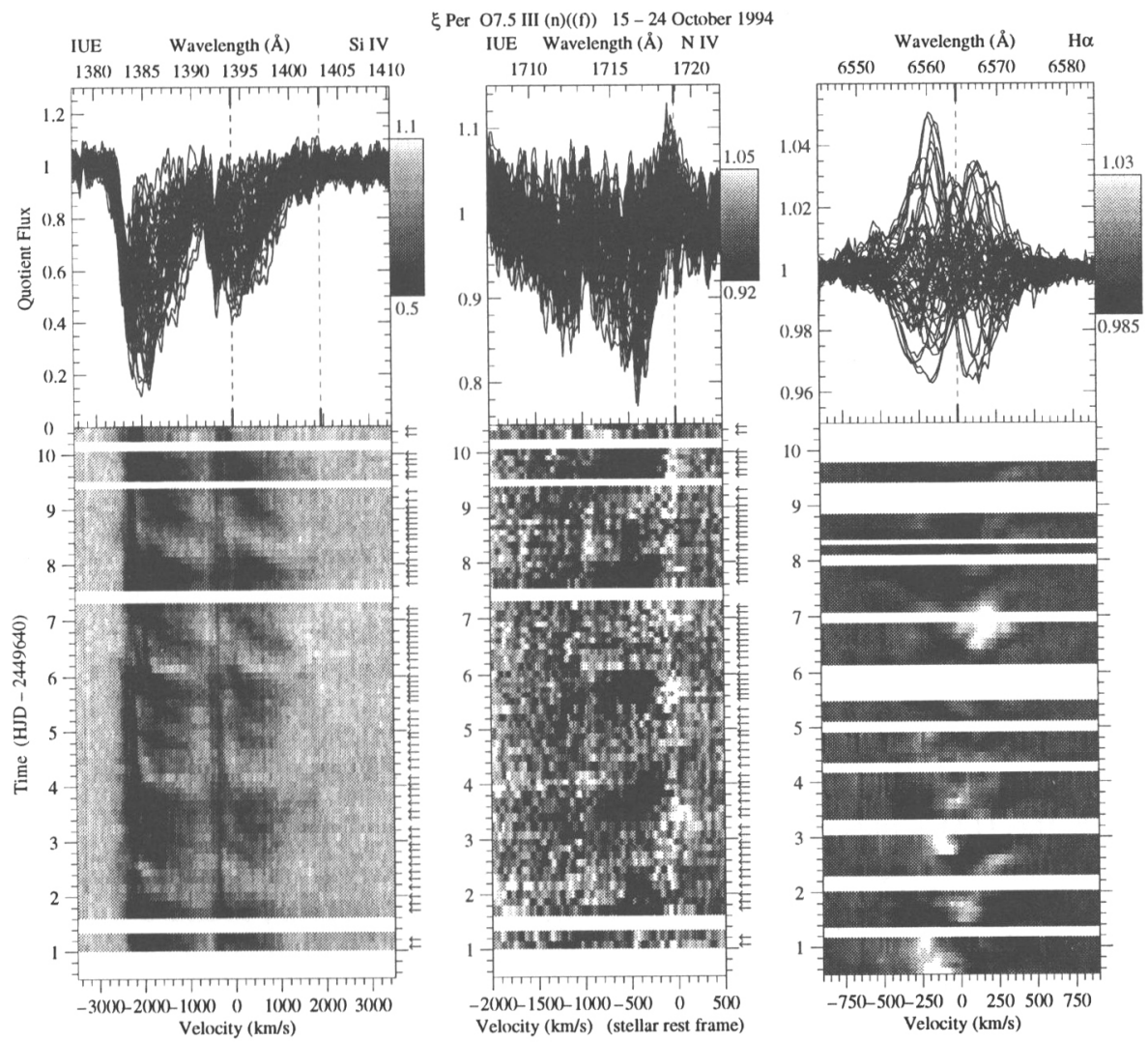

Fig. 1. Dynamic quotient spectra showing the variability of the ultraviolet Si IV resonance doublet and subordinate N IV line, and the optical $\mathrm{H} \alpha$ line in the O7.5III star $\xi$ Per. The UV and optical data were taken simultaneously during a period of more than 10 days of continuous observations. The N IV line indicates the appearance of a strong DAC, whose subsequent evolution is registered by the Si IV doublet. Also the $\mathrm{H} \alpha$ line exhibits a clear increase in absorption at low blue-shifted velocities down in the base of the wind. Close to line center the variations are much more complicated due to projection effects, a varying amount of incipient wind emission, and photospheric variations (from Henrichs et al. 1998).

As is found to be true in several $O$ stars, the DACs reach a maximum column density at a velocity of about $0.75 v_{\infty}$ and subsequently fade in strength. When DACs are measured in more than one resonance doublet, consistent results are obtained, supporting the interpretation that DACs are due to changes in wind density and/or velocity rather than changes in the ionization structure of the stellar wind. The change in $N_{\text {col }}$ suggests that the 
covering factor of the absorbing material in the line of sight changes as a function of distance from the star.

A key issue is the recurrence timescale of DACs; they repeat on a timescale comparable to the estimated stellar rotation period. Fourier analyses performed on the datasets clearly reveal this periodicity (Kaper et al. 1998a). In Tab. 1 the observed "wind periods" (corresponding to the DAC recurrence timescales) are listed for a sample of O-type stars. The stars are ordered according to the estimated maximum rotation period:

$$
P_{\max }=50.6(v \sin i)^{-1}\left(\frac{R_{\star}}{R_{\odot}}\right) \text { days, }
$$

where $R_{\star}$ is the stellar radius. The table shows that stars with a shorter DAC cycle end up higher on the list, consistent with the interpretation that this cycle relates to the rotation period of the star.

\section{Coordinated $\mathrm{H} \alpha$ monitoring}

Also subordinate lines, formed relatively close to the star, show signatures of wind variability. Prinja et al. (1992) and Henrichs et al. (1994) demonstrated that for some stars DACs appear as well in the N IV line at $1718 \AA$, in concert with the DACs observed in the UV resonance lines (Fig. 1). Strong subordinate lines are present in the optical spectrum as well (e.g. He I $5876 \AA$, $\mathrm{H} \alpha$ ). In his survey of a dozen OB supergiants Ebbets (1982) found dramatic changes in shape and strength of $\mathrm{H} \alpha$, but the time sampling of his observations was too irregular to permit the timescales (1-10 days) to be estimated reliably. Kaper et al. (1997) obtained coordinated UV and $\mathrm{H} \alpha$ observations for a number of bright $\mathrm{O}$-type stars and demonstrated that the $\mathrm{H} \alpha$ variability is directly linked to the DAC behaviour in the UV resonance lines. The $\mathrm{H} \alpha$ line yields the same wind period (Tab. 1) and can thus be used to measure the cyclical appearance of DACs in UV resonance lines.

An extensive campaign of coordinated UV and optical observations of the O7.5III star $\xi$ Per was carried out in October 1994 (Henrichs et al. 1998). The wind period of 2 days is very apparent in both the resonance and the subordinate lines; the strong DACs in the Si IV doublet are accompanied by phases of enhanced blue-shifted absorption at low velocities in the N IV and $\mathrm{H} \alpha$ lines. The $\mathrm{H} \alpha$ observations were obtained at 7 different observatories spread over the northern hemisphere. The core of the $\mathrm{H} \alpha$ line shows much more complicated variations, probably because of varying amounts of wind emission (also from the regions outside the line of sight) and variations deep down in the photosphere (De Jong 1998).

\section{H $\alpha$ campaign}

In order to test the hypothesis that $\mathrm{O}$-star winds are modulated by the rotation of the underlying star, we monitored a large sample of $\mathrm{O}$ stars (38) for 
Table 1. Measured "wind" period in O star spectra compared to the estimated maximum rotation period (based on the stellar parameters listed by Howarth \& Prinja (1989) and references therein). Runaway stars are indicated by (R). References: 1) Kaper et al. 1998a; 2) Prinja et al. 1992; 3) Howarth et al. 1993; 4) Fullerton et al. 1992: from He I $5876 \AA$; 5) Prinja 1988 ; 6) Stahl et al. 1996; 7) Kaper et al. 1997; 8) Kaper et al. 1998b.

\begin{tabular}{|c|c|c|c|c|c|c|c|}
\hline Name & $\begin{array}{l}\text { Sp. Type } \\
\text { (Walborn) }\end{array}$ & $\begin{array}{c}v \sin i \\
\left(\mathrm{~km} \mathrm{~s}^{-1}\right)\end{array}$ & $\begin{array}{c}R \\
\left(R_{\odot}\right)\end{array}$ & $\begin{array}{c}P_{\max } \\
\text { (days) }\end{array}$ & $\begin{array}{c}P_{\text {wind }} \\
\text { UV }\end{array}$ & $\begin{array}{c}\text { (days) } \\
\mathrm{H} \alpha\end{array}$ & Reference \\
\hline$\zeta \mathrm{Oph}(\mathrm{R})$ & $09.5 \mathrm{~V}$ & 351 & 8 & 1.2 & 0.9 & & 3 \\
\hline $68 \mathrm{Cyg}$ (R) & O7.5 III:n((f)) & 274 & 14 & 2.6 & 1.4 & 1.3 & 1,7 \\
\hline$\xi \operatorname{Per}(R)$ & O7.5 III(n)((f)) & 200 & 11 & 2.8 & 2.0 & 2.0 & 1,7 \\
\hline$\lambda$ Cep (R) & O6 I(n)fp & 214 & 19 & 4.5 & 1.3 & 1.2 & 1,7 \\
\hline$\zeta \operatorname{Pup}(\mathrm{R})$ & O4 I(n)f & 208 & 19 & 4.6 & 0.8 & 0.9 & 2,8 \\
\hline HD 34656 & O7 II(f) & 106 & 10 & 4.8 & 1.1 & & 1 \\
\hline 15 Mon & O7 V $((f))$ & 63 & 10 & 8.0 & $>4.5$ & & 1 \\
\hline $63 \mathrm{Oph}$ & O7.5 II ((f)) & 80 & 16 & 10.2 & $>3$ & & 5 \\
\hline HD 135591 & O7.5 $\operatorname{III}((\mathbf{f}))$ & 65 & 14 & 10.9 & & 3.1 & 8 \\
\hline$\lambda$ Ori A & O8 III((f)) & 53 & 12 & 11.5 & $\sim 4$ & 2.0: & 1 \\
\hline$\theta^{1}$ Ori C & O6-O4 var & 50: & 12: & $12:$ & "15.4" & 15.4 & 6 \\
\hline 19 Cep & O9.5 Ib & 75 & 18 & 12.1 & 4.5 & $\sim 5$ & 1,7 \\
\hline$\mu$ Nor & O9.7 Iab & 85 & 21 & 12.5 & & 6.0 & 8 \\
\hline$\alpha \operatorname{Cam}(\mathrm{R})$ & O9.5 Ia & 85 & 22 & 13.2 & & 5.6 & 8 \\
\hline$\zeta$ Ori A & O9.7 Ib & 110 & 31 & 14.3 & $\sim 6$ & 6 & 1,8 \\
\hline $10 \mathrm{Lac}$ & $\mathrm{O} 9 \mathrm{~V}$ & 32 & 9 & 15.3 & $\sim 7$ & & 1 \\
\hline HD $112244(\mathrm{R})$ & O8.5 Ib(f) & 70 & 26 & 18.8 & & 6.2 & 8 \\
\hline HD $57682(\mathrm{R})$ & O9 IV & 17 & 10 & 29.8 & & $>6$ & 8 \\
\hline HD 151804 & O8 Iaf & 50 & 35 & 35.4 & & $2-3,7.3$ & 4,8 \\
\hline
\end{tabular}

$\mathrm{H} \alpha$-profile variations (Kaper et al. 1998b). Most (75\%) of the monitored O stars, excluding the known short-period spectroscopic binaries, exhibit line profile variations in $\mathrm{H} \alpha$. Fig. 2 presents the amplitude of $\mathrm{H} \alpha$ variability (expressed as the intergral of the time variance spectrum (TVS), Fullerton et al. 1996) as a function of $\mathrm{H} \alpha$ equivalent width. The open symbols correspond to spectroscopic binaries, the closed symbols are single stars. The main-sequence stars (circles) do not show any indication of (wind) variability, though many of them are known to have variable DACs in their UV resonance lines. This is probably due to the low density of their winds causing any variations to remain below our detection threshold. The main-sequence binaries have a strong TVS spectrum, because the intrinsically constant line profile is moving back and forth due to orbital motion. With increasing luminosity (triangles: giants; squares: supergiants) the $\mathrm{H} \alpha$ equivalent width gets more negative (incipient emission due to stellar wind) and the variability amplitude gets larger. This indicates that the variability in the $\mathrm{H} \alpha$ line is mainly due to variations 


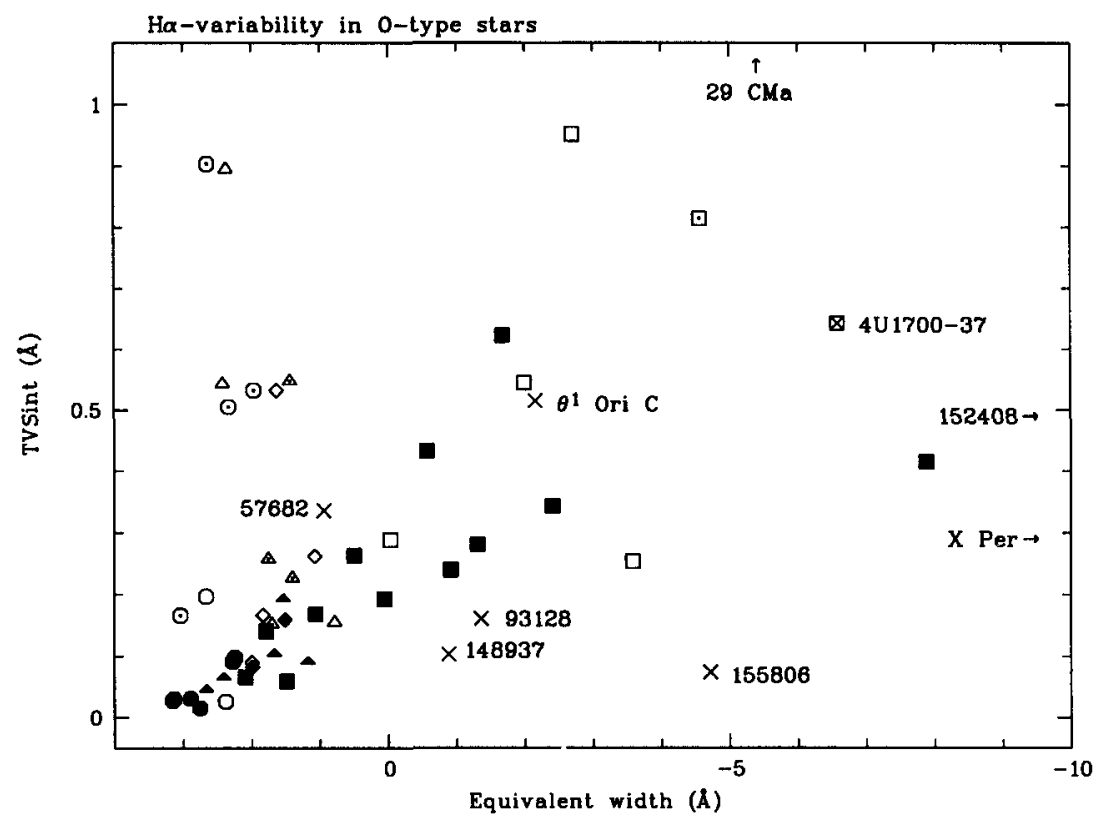

Fig. 2. The amplitude of $\mathrm{H} \alpha$ variability shown as a function of $\mathrm{H} \alpha$ equivalent width. The single main-sequence stars (closed circles) do not exhibit wind variability. The spectroscopic binaries (open symbols) have relatively large variability amplitudes, because of the radial-velocity variations resulting from orbital motion. The $\mathrm{H} \alpha$ profiles of stars with stronger stellar winds (triangles: giants; squares: supergiants) have a larger contribution of wind emission (negative EW) and a larger variability amplitude. The main-sequence stars $\theta^{1}$ Ori C and HD57682 have strongly variable $\mathrm{H} \alpha$ emission. The largest variability amplitude is observed for the SB2 $29 \mathrm{CMa}$ which includes a strong shock resulting from the stellar-wind collision.

in the stellar wind which are more easily detected in strong stellar winds. In close, massive binary systems the collision of the two stellar winds can result in an additional variability component (Thaller 1997).

For $41 \%$ of the single $\mathrm{H} \alpha$-variables a wind period could be determined (Tab. 1), which is in all cases consistent with (an integral fraction of) the expected stellar rotation period. For those $\mathrm{O}$ stars we monitored in $\mathrm{H} \alpha$ and for which a DAC recurrence timescale is known, the UV and $\mathrm{H} \alpha$ wind periods are equal (within the measurement accuracy).

\section{On the origin of cyclical wind variability}

Several models have been put forward to explain the observed properties of DACs. The cyclical nature of wind variability indicates that stellar rotation 
must play an important role. Most promising is the CIR model proposed by Mullan $(1984,1986)$, recently worked out by Cranmer \& Owocki (1996). In this model, structure in the stellar wind is caused by the interaction of fast and slow streams that originate at neighbouring locations on the stellar surface. Due to the rotation of the star the streams are curved, causing fast wind material to collide with slow material in front. The interaction region has a spiral shape and corotates with the star, though not the wind material itself which mainly flows in the radial direction. Prinja \& Howarth (1988) suggested such a spiral-shaped, large-scale structure to be present in the wind of 68 Cyg. The coordinated UV and $\mathrm{H} \alpha$ observations in Kaper et al. (1997) strongly support the CIR model. Comparison between model and observations suggests that wind streams with relatively slow speed interact with a faster ambient wind, rather than high-speed streams in a slow wind (Cranmer \& Owocki 1996). Therefore, $v_{\text {asymp }}$ might be somewhat lower than the $v_{\infty}$ of the ambient wind. The systematic difference in $v_{\text {asymp }}$ measured in some stars could be due to a difference in aspect angle of the CIR, or to a difference in contrast (velocity difference fast and slow wind), or both.

In order to work, the CIR model needs a certain structure imposed at the stellar surface to produce flows with different kinematic properties. Two candidate physical mechanisms are: (i) non-radial pulsations (NRP), or (ii) surface magnetic fields. NRP are detected in several $\mathrm{O}$ stars (Fullerton et al. 1996, Henrichs, this volume). De Jong et al. (1998) have recently shown that also $\xi$ Per (Fig. 1) is a non-radial pulsator. The period and corresponding "pattern speed" of the NRP are, however, not consistent with the observed wind cycle of 2 or 4 days. Our working hypothesis is that the outflow properties at the base of the wind are influenced by a magnetic field anchored in the star. The presence of surface magnetic fields is difficult to prove with direct observational methods (Mathys, this volume). Spectropolarimetric observations to detect a surface magnetic field in $\xi$ Per were not conclusive (Henrichs et al. 1998) and resulted in an upper limit of $70 \mathrm{G}$. The search for the photospheric connection is the subject of current investigations.

\section{References}

Cranmer, S.R., Owocki, S.P. 1996, ApJ 462, 469

De Jong, J.A. 1998, in Proc. ESO workshop on Cyclical Variability in Stellar Winds, Eds. Kaper \& Fullerton, p. 336

De Jong, J.A., Henrichs, H.F., Schrijvers, C., et al. 1998, submitted to A\&A

Ebbets, D. 1982, ApJS 48, 399

Fullerton, A.W., Gies, D.R., Bolton, C.T. 1992, ApJ 390, 650

Fullerton, A.W., Gies, D.R., Bolton, C.T. 1996, ApJS 103, 475

Henrichs, H.F., Kaper, L., Nichols, J. 1994, A\&A 285, 565

Henrichs, H.F., De Jong, J.A., Nichols, J, et al. 1998, in Proc. "Ultraviolet astrophysics beyond the IUE final archive", Eds. Wamsteker, González Riestra, Harris (ESA SP-413), p. 157 
Howarth, I.D., Prinja R. 1989, ApJS 69, 527

Howarth, I.D., Bolton, C.T., Crowe, R.A., et al., 1993, ApJ 417, 338

Kaper, L., Henrichs, H.F., Nichols, J., et al. 1996, A\&A Supp. Ser. 116, 1

Kaper, L., Henrichs, H.F., Fullerton, A.W., et al., 1997, A\&A 327, 281

Kaper, L., Henrichs, H.F., Nichols, J., Telting, J.H. 1998a, submitted to A\&A

Kaper, L., Fullerton, A.W., Baade, D., et al. 1998b, in preparation

Massa, D., Fullerton, A.W., Nichols, J.S., et al. 1995, ApJ, 452, L53

Mullan, D.J. 1984, ApJ 283, 303

Mullan, D.J. 1986, A\&A 165, 157

Prinja, R.K. 1988, MNRAS 231, 21P

Prinja, R.K., Howarth, I.D. 1988, MNRAS 233, 123

Prinja, R.K, Balona, L.A., Bolton, C.T. et al. 1992, ApJ 390, 266

Stahl, O., Kaufer, A., Rivinius, Th., et al. 1996, A\&A 312, 539

Thaller, M. 1997, ApJ 487, 380

\section{Discussion}

Eversberg: Could the different terminal velocities for DACs be due to temperature differences on the stellar surface?

Kaper: It might well be that the velocity contrast between fast and slow streams in the wind is different at different areas above the stellar surface, resulting in different values for $v_{\text {asymp }}$. In the models of Cranmer \& Owocki (1996), the difference in outflow properties is due to a difference in luminosity (bright spot).

Moffat: Do you have any constraints on the lifetime of the spot or whatever it is on the surface that causes DACs?

Kaper: Our long-term studies show that the characteristic DAC pattern is clearly identifiable in all our datasets obtained over a period of several years. Detailed differences are found from year to year, suggesting a long-term variation on the order of months.

Verdugo: You've said that the mechanism producing the DACs is close to the stellar surface in the region where $\mathrm{H} \alpha$ is formed. Then, how do you explain that stars which don't show any variability in the $\mathrm{H} \alpha$ line present DACs in their spectra?

Kaper: I think this is due to the low density of the winds of main-sequence stars. The $\mathrm{H} \alpha$ line strength is proportional to $\rho^{2}$, while the strength of the resonance lines showing the DACs is proportional to $\rho$. 